\title{
BMJ Open Rest Evaluation for Active Concussion Treatment (ReAct) Protocol: a prospective cohort study of levels of physical and cognitive rest after youth sports-related concussion
}

Jingzhen Yang, ${ }^{1,2}$ Keith Yeates, ${ }^{\circledR}{ }^{3}$ Lindsay Sullivan, ${ }^{1}$ Bhavna Singichetti, ${ }^{1}$ Alison Newton, ${ }^{1}$ Pengcheng Xun, ${ }^{4}$ H Gerry Taylor, ${ }^{2,5}$ James MacDonald, ${ }^{2,6}$ Thomas Pommering, ${ }^{2,6}$ Michael Tiso, ${ }^{7}$ Daniel Cohen, ${ }^{2,8}$ Yungui Huang, ${ }^{9}$ Jeremy Patterson, ${ }^{9}$ Zhong-lin $\mathrm{Lu}^{10}$

To cite: Yang J, Yeates $\mathrm{K}$, Sullivan L, et al. Rest Evaluation for Active Concussion Treatment (ReAct) Protocol: a prospective cohort study of levels of physical and cognitive rest after youth sports-related concussion. BMJ Open 2019;9:e028386. doi:10.1136/ bmjopen-2018-028386

- Prepublication history and additional material for this paper are available online. To view these files, please visit the journal online (http://dx.doi. org/10.1136/bmjopen-2018028386).

Received 12 December 2018 Revised 14 February 2019 Accepted 11 March 2019

\section{Check for updates}

(C) Author(s) (or their employer(s)) 2019. Re-use permitted under CC BY-NC. No commercial re-use. See rights and permissions. Published by BMJ.

For numbered affiliations see end of article.

Correspondence to Dr Jingzhen Yang;

Ginger.Yang@

nationwidechildrens.org

\section{ABSTRACT}

Introduction Although current guidelines for the early clinical management of sports-related concussion (SRC) call for a gradual return-to-activity, the optimal level of rest needed to promote recovery remains unknown. This paper describes the protocol of the Rest Evaluation for Active Concussion Treatment (ReAct) study which objectively measures physical and cognitive rest following SRC and its relation to recovery among youth athletes.

Methods and analysis Youth athletes aged 11-17 years are recruited preinjury and enrolled within 72 hours following a physician-diagnosed concussion. Injury information and acute clinical presentation are assessed at the time of injury. Youth participants are prospectively followed to objectively monitor daily physical and cognitive rest using two electronic devices: ActiGraph (to measure physical rest and sleep) and Narrative Clip (to measure cognitive rest), along with self-reported postconcussive symptoms using daily surveys. Other concussion outcomes, including functional outcomes, are assessed by surveying youth and their parents at three time points: (1) within 72 hours of injury, (2) at day 7 postenrolment and (3) at symptom resolution (or a maximum of 45 days postconcussion).

Ethics and dissemination This study has received ethical approval from the Institutional Review Board (IRB) at the participating institution (IRB at Nationwide Children's Hospital, IRB16-00613). The results of the study will be presented at national and international scientific conferences and published in peer-reviewed journals.

\section{INTRODUCTION}

Sports-related concussion (SRC) is a major public health problem in the United States, affecting approximately two million children each year. ${ }^{1-3}$ Concussions can temporarily disrupt brain function and result in detrimental effects on youths' developing brains, which may affect their short- and

\section{Strengths and limitations of this study}

- This study fills critical knowledge gaps by objectively monitoring both physical and cognitive rest/activity among youth following a sports-related concussion.

- The study design allows for the gathering of data on physical and cognitive rest and other study measures within 72 hours of injury.

- Cutting-edge computer techniques are used to match the data collected by an ActiGraph and Narrative Clip, which minimises data processing time.

- Quantifying cognitive rest is challenging, although data on cognitive activities are documented.

- The cognitive activities participants engage in during school hours are not measured due to privacy concerns of wearing the Narrative Clip at school.

long-term physical, cognitive, emotional and sleep health. ${ }^{4-6}$ In most children, concussion symptoms resolve within one to 3 weeks postinjury. ${ }^{6}$ However, evidence suggests that up to one-third of concussed children exhibit persistent postconcussion symptoms or other functional impairments which can last months to years after injury. ${ }^{7-10}$ Current guidelines for the early clinical management of SRC call for a brief period of rest during the acute phase (24-48 hours) postinjury, followed by a gradual and progressive increase in activities while staying below cognitive and physical symptom-exacerbation thresholds (ie, activity level should not bring on or worsen symptoms). ${ }^{11-13}$ However, these guidelines are not strongly evidence-based.

Animal models of concussion have shown that a period of rest immediately following concussion is beneficial as it reduces physical 
and cognitive demands and frees up energy for significantly increased metabolic needs during recovery. ${ }^{14-18}$ Evidence from humans suggests that engaging in high levels of physical activity prematurely after a concussion can exacerbate symptoms, increase symptom duration and lead to greater neurocognitive and functional impairments. ${ }^{19-23}$ However, emerging research has begun to highlight the potential benefits of both moderate physical and cognitive activity postconcussion. ${ }^{24-27} \mathrm{~A}$ recent randomised controlled trial (RCT) of 88 individuals with concussion (aged 11-22 years) treated in a paediatric emergency department (ED) showed that 5 days of prescribed strict rest did not offer additional benefits compared with prescribed standard care. ${ }^{28}$ In fact, Thomas and colleagues (2015) found that 5 days of 'complete' rest (ie, avoidance of all physical and cognitive activities) following a concussion could exacerbate symptoms. ${ }^{28}$ Others have reported that a period of 'complete rest' postconcussion may be impractical or unnecessary for some individuals. ${ }^{28-30}$ However, the optimal level and timing of postinjury physical and cognitive rest needed to promote recovery is unknown. ${ }^{9} 11$

Previous studies that measured physical and cognitive rest postconcussion have been limited by the use of self-report data. ${ }^{19-22}$ Some studies have only measured prescribed rest while others have failed to distinguish between physical and cognitive rest. ${ }^{19-22}$ The definition of physical and cognitive rest used in previous studies has also varied widely, from complete rest to decreased duration and frequency of cognitive and physical activities. ${ }^{19-21}$ A comprehensive, prospective study using objective measures is necessary to identify the optimal level of physical and cognitive rest for youth after SRC, so that individually tailored and effective care can be provided to these youth. ${ }^{31-33}$

\section{Objectives}

The aims of the Rest Evaluation for Active Concussion Treatment (ReAct) study are to: (1) objectively measure the levels of physical and cognitive rest following SRC, (2) describe levels of physical and cognitive rest in relation to postconcussion symptoms and functional outcomes (ie, functional disability and quality of life) and (3) examine such relationship among youth with high- versus low-risk concussions (defined based on initial symptom and likelihood of prolonged recovery) ${ }^{3435}$ We hypothesise that the relationship between levels of physical and cognitive rest and postconcussion symptoms and functional outcomes will be curvilinear (eg, U-shaped), such that youth engaging in optimal levels of physical and cognitive rest following SRC will experience less severe postconcussion symptoms and functional outcomes as compared with youth whose level of rest is below or above the optimal level. We also hypothesise that postinjury levels of physical and cognitive rest will be more strongly related to postconcussion symptoms and functional outcomes among youth with high-risk concussions than among those with low-risk concussions. This paper is a description of the methods to address these hypotheses.

\section{METHODS}

\section{Study design and overview}

The ReAct study uses a prospective cohort design, with repeated measures. Eligible youth aged 11-17 years and a parent/legal guardian ('parent') are invited to participate in the study either preinjury during preseason team meetings or following a concussion. Youth and parents ('dyads') are screened for study eligibility following their physician-confirmed SRC, and then enrolled after providing written assent and consent, respectively (figure 1).

Injury information and acute clinical presentation (signs and symptoms, mental status, balance, neuropsychological function) are assessed at the time of injury or at the time of the initial clinic visit as part of routine clinical care. Participants are followed throughout their recovery by a trained research team member ('researcher'), and complete assessments of postconcussion symptoms, functional outcomes and other study outcomes (eg, quality of life) at three time points: (1) within 72 hours of injury (assessment 1), (2) 7 days after enrolment (assessment 2) and (3) at symptom resolution or 45 days postinjury (assessment 3), whichever occurs first (figure 1). Symptom resolution is defined as being symptom-free or as having symptoms return to premorbid levels as confirmed by a certified athletic trainer (AT) or physician. Physical and cognitive rest (the inverse of activity) are monitored for a 7-day period following the first assessment using two electronic devices: an ActiGraph and a Narrative Clip. The ActiGraph (with an accompanied heart rate sensor) records 24 hours physical activity (eg, steps) and sleep cycles. The Narrative Clip records daytime activity (outside of school time) by taking a photo every $30 \mathrm{~s}$, allowing us to code their cognitive activities. Additionally, youth participants complete a hard-copy or online daily survey, which assesses their physical and cognitive activities, postconcussion symptoms and pain throughout their enrolment in the study.

The first assessment lasts about 1 hour, and the two follow-up assessments last about $30 \mathrm{~min}$ each. Most measures, described below, are drawn from the National Institutes of Health Common Data Elements for Paediatric Traumatic Brain Injury and Sports-related Studies. ${ }^{36}$ The study began on 1 September 2016 and will end on 31 August 2019.

\section{Study population}

Youth aged 11-17 years must have sustained a direct or indirect force(s) to the brain during an organised or recreational sport activity, and received a physician-confirmed diagnosis of concussion. ${ }^{11} 37$ The dyad must be able to meet a researcher within 72 hours of injury.

Youth are excluded if any of the following apply: (1) the concussion occurred during participation in a 


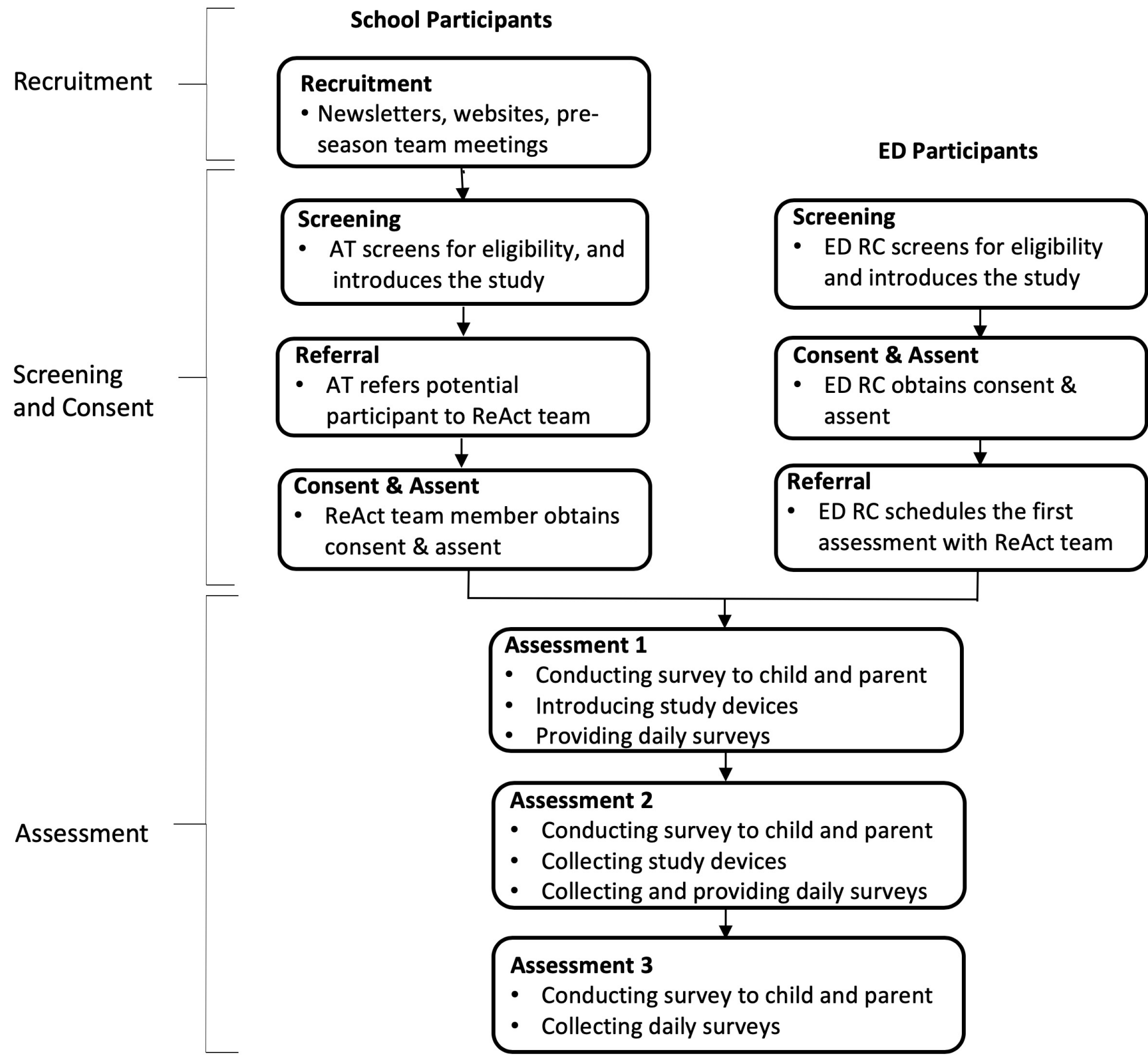

Figure 1. Overview of Study Procedures

Figure 1 Overview of study procedures. AT, athletic trainer; ED, emergency department; RC, research coordinator; ReAct, Rest Evaluation for Active Concussion Treatment.

non-sports-related activity (eg, motor vehicle crash, fall), (2) individuals with multiple trauma (eg, face, neck injury, broken bone(s)), (3) the concussion involved a penetrating injury, (4) the child requires neurosurgical intervention or hospital admission, (5) the injury was associated with illicit drug or alcohol use, (6) the child has an associated injury that is likely to interfere with neuropsychological testing (eg, injury that affects eyesight) or (7) the participant is physically unable to wear the study devices. Designated physicians review potential participants' information to ensure that they meet the study eligibility criteria.
Study procedures

Recruitment and consent

Participants recruited from middle schools and high schools

Youth are identified at three middle schools and 15 high schools, all of which have contracts for athletic training services with Nationwide Children's Hospital (NCH). All ATs in these schools are NCH employees and some also work at NCH concussion clinics.

ReAct study information (ie, study email address, phone number, other contact information) is distributed via participating schools' newsletters, on NCH's and participating schools' websites, and at preseason 
team and parent meetings. At preseason team and parent meetings, our research team presents a brief overview of the study to students, parents and coaches. Soon after a physician-confirmed diagnosis of concussion, school ATs, who have been trained on study protocols, reintroduce the study to potential participants and then notify our research team if the family is interested. A researcher then contacts the family to confirm eligibility, and schedules an in-person meeting with the concussed youth and a parent within 72 hours of injury at a location that is convenient for the child and parent (eg, clinic, participant's home, school or library). During this initial meeting, the researcher answers any questions, obtains informed written consent and assent from the youth and parent, respectively, and completes enrolment procedures and the first assessment.

\section{Participants recruited from ED}

Prospective participants are identified from youth who present to the ED at $\mathrm{NCH}$ and receive a physician-confirmed diagnosis of SRC. ED recruitment started in 25 April 2017 to help increase enrolment of study participants within 72 hours of injury. Soon after a physician-confirmed diagnosis of SRC, an ED clinical research coordinator, who has been trained on study protocols, screens for eligibility and introduces the study to eligible participants. The ED clinical research coordinator obtains written informed consent and assent from those interested in participating, completes enrolment procedures, schedules the first assessment within 72 hours of injury and provides the research team with the contact information of the enrolled participant and their preferred method of contact via a secure email. A member of the ReAct research team then contacts the family to confirm the date, time and location of the first assessment.

\section{Data collection}

During the first assessment, conducted in-person within 72 hours of injury, the researcher administers the first survey to the youth and the parent. The survey asks about demographic information of the dyad, postinjury concussion symptoms and functional outcomes of the youth, and postinjury events (eg, return to school, return to play $[\mathrm{RTP}]$ ). In addition, the parent is asked to retrospectively rate the premorbid functioning of the youth.

Following the completion of the survey, the family is provided with the two study devices (Narrative Clip and ActiGraph with the accompanied heart rate sensor, see description below) and a laptop computer. The researcher activates the devices and shows the dyad how to use them. The ActiGraph is initialised based on the participating youth's height, weight, age and preferences for wearing the device (ie, wrist vs ankle, left vs right side, dominant vs non-dominant hand/ foot). After the ActiGraph is initialised, it automatically connects to the heart rate sensor (which the participant is instructed to wear around their torso) via Bluetooth. The youth participant is instructed to wear these waterproof devices 24 hours a day for the next 7 days. Next, the Narrative Clip and its use are explained to the participating youth and his/her parent. Specifically, the youth participant and the parent are shown how to wear the Narrative Clip with the custom-designed headband, and how to connect the Narrative Clip to the laptop via a universal serial bus (USB) cable. The youth participant and the parent are instructed to do this each night. Once the Narrative Clip is connected to the laptop, the data (ie, photographs) are automatically uploaded to the computer and the Narrative Clip begins to charge. Additionally, the youth is instructed to complete a daily survey each night, either online via REDCap or a pen-and-paper survey, based on his/her personal preference. The daily surveys include questions about activities related to the sport in which the injured youth is returning to soon after recovery, as well as physical and cognitive rest and postconcussion symptoms. At the end of the first assessment, the youth participant and parent receive detailed written instructions regarding their daily study responsibilities and the contact information of the researcher if they have questions. A researcher contacts the family within 48 hours of the first assessment to see how the participant is feeling and to ensure device use is going smoothly.

Seven days after the first assessment, the researcher meets with the participating family to complete the second assessment. During this assessment, the dyad completes a survey, with similar questions to the first survey, and returns the study devices. The youth participant is instructed to continue completing daily surveys until symptom resolution. After data are downloaded, the study devices are reset (wiped of all data and photographs) by a designated information technology professional at $\mathrm{NCH}$, sanitised and made available for future participants.

Once the youth's symptoms resolve or they reach 45 days postinjury, whichever occurs first, the dyad complete the third and final assessment that includes the completion of a survey with similar questions as the two previous surveys.

To ensure data quality and participant compliance, the researcher frequently checks-in with the participating family to ensure that there are no problems with completing the daily surveys and the uploading of photographs to the computer. The daily surveys also include a prompt for participants to upload the data from the Narrative Clip to the computer. To encourage participant compliance, participants are compensated (eg, gift cards) for completing assessments, device usage, photograph upload and daily survey completion.

\section{Study variables and measures}

Study variables and measures included in this study organised by the data source, time and method of data collection are summarised in table 1 . 


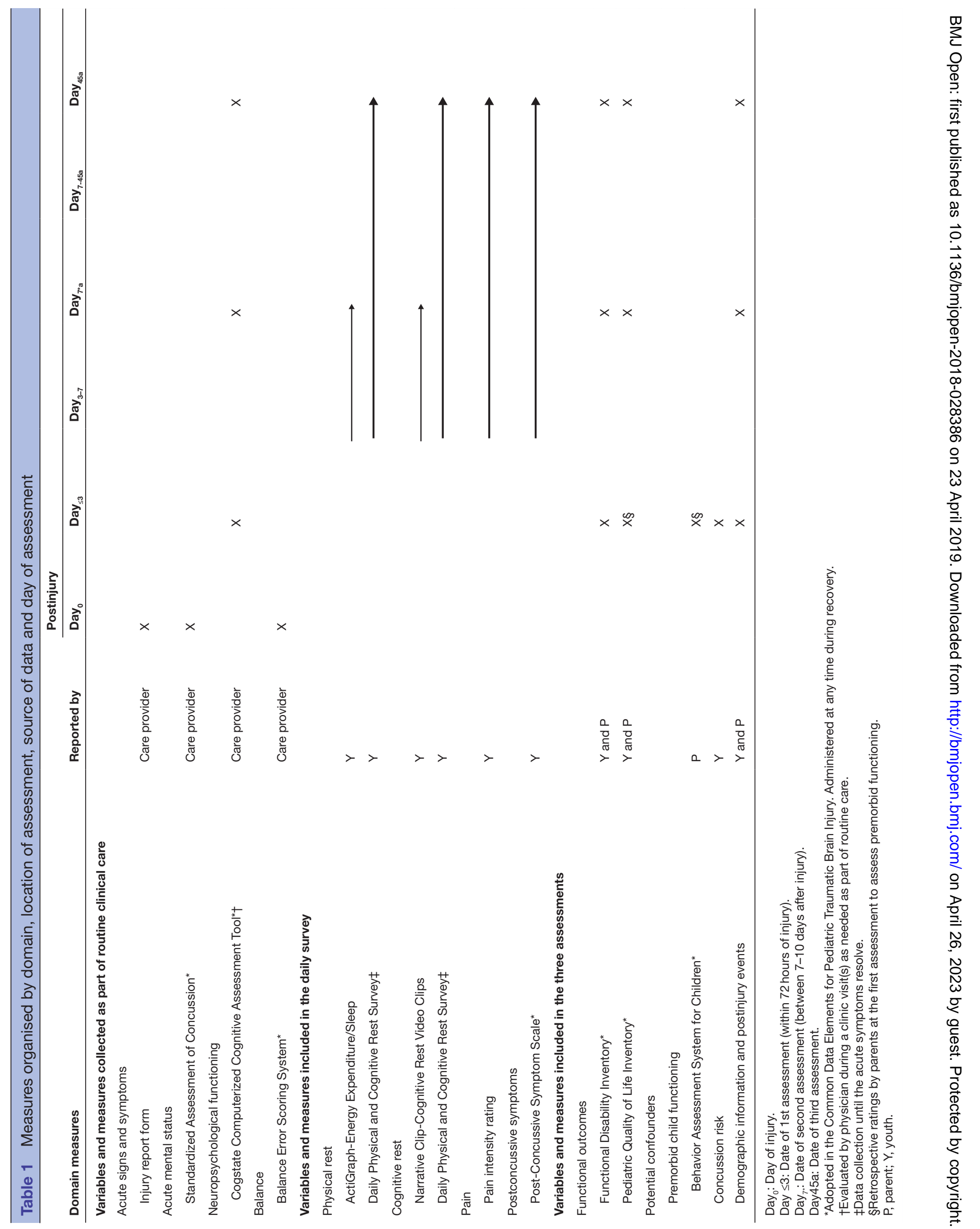


Variables and measures collected as part of routine clinical care

Acute signs and symptoms

Acute signs and symptoms are measured using a standardised injury report form completed by the certified AT or ED physician. Injury details and acute signs and symptoms (ie, loss of consciousness, post-traumatic amnesia, neurological status and other clinical features) are collected and verified by an attending physician.

\section{Acute mental status}

The Standardized Assessment of Concussion (SAC), used to measure acute mental status, ${ }^{38}{ }^{39}$ is completed by the certified AT or an attending physician. The SAC provides a rapid assessment of orientation, attention and immediate and delayed memory, as well as acute postconcussion symptoms. SAC has been used extensively in research on SRCs in youth. ${ }^{39} 40$

\section{Neuropsychological functioning}

Neuropsychological functioning is evaluated by an attending physician using Cogstate Computerized Cognitive Assessment Tool (CCAT), which uses computerised card games to test cognitive ability. ${ }^{41-43}$ The CCAT is currently used at the seven $\mathrm{NCH}$ concussion clinics as part of routine clinical care. It measures four simple tasks with standard scores $(\mathrm{M}=100, \mathrm{SD}=10)$ : (1) processing speed, (2) attention, (3) learning and (4) working memory. The CCAT has been validated among youth and has excellent psychometric characteristics. ${ }^{43}$ The CCAT data collected by the NCH concussion clinics as part of routine clinical care will be available to the study team. Baseline data is also available via prior health records.

\section{Balance}

Balance is assessed by the certified AT or an attending physician using the Balance Error Scoring System (BESS), a widely used tool. ${ }^{44}$ The BESS has participants perform three stances (narrow double leg, single leg and tandem) on two footing surfaces (firm surface/floor vs medium density foam). Each stance is held, with hands on hips and eyes closed, for a period of $20 \mathrm{~s}$. The errors (eg, opening eyes, lifting hands off hips or stumbling) committed during each condition are counted and recorded. The BESS has shown satisfactory reliability in youth and adolescents. ${ }^{44}$

\section{Variables and measures included in daily survey Physical rest}

Physical rest is defined as not engaging in physical activity beyond activities of daily living. ${ }^{14}$ In this study, we measure the duration and quality of sleep, and the duration and intensity of physical activity, using an ActiGraph (Model GT3XP-BT), which is a $4.6 \times 3.3 \times 1.5 \mathrm{~cm}$, validated, non-invasive, wrist-watch-like device with a Bluetooth-paired strap that records heart rate ('ActiGraph'). ${ }^{45}$ The ActiGraph continually measures rest cycles with an Actimetry sensor. The device can store up to $2 \mathrm{~GB}$ of data for up to 25 days. Actigraphy has been shown to provide accurate biometric data, including energy expenditure, activity intensity, heart rate, sleep duration and sleep efficiency. ${ }^{45}$ In this study, Actigraphy provides data on sleep duration (number of sleep hours) and sleep quality (the ratio of deep- to light-sleep). Actigraphy also provides data on the intensity of the physical activities in which the youth participant engages, expressed as the ratio of energy expenditure to basal energy expenditure (number of calories burned in the resting state), adjusting for participant age, sex, height and weight. ${ }^{46}$ Actigraph has been used in monitoring physical activity and sleep in youth following concussion. ${ }^{47} 48$ In this study, the level of physical rest for each day is measured as the inverse of the participant's summary score, which is scored as the total duration of various physical activities multiplied by the corresponding intensity of these physical activities (duration $\mathrm{x}$ intensity) across a whole day. Additionally, self-reported physical rest data, including sport activity specific to the participant's sport, are collected via the daily surveys throughout the participant's enrolment.

\section{Cognitive rest}

Cognitive rest is defined as not engaging in activities requiring attention or concentration. ${ }^{14}$ In this study, cognitive rest is monitored using a Narrative Clip which is a $36 \times 36 \times 9 \mathrm{~mm}$ wearable camera weighing $20 \mathrm{~g}$. ${ }^{49}$ The camera silently and automatically captures high-resolution digital images every $30 \mathrm{~s}$. This device can store up to 4000 digital images, and has a 2-day battery life.

Participants wear the Narrative Clip for a 7-day period following enrolment. They are asked to wear the Narrative Clip as much as possible during this 7-day period. To ensure the Narrative Clip captures a normal visual field, a three-dimensional (3D) printed wedge (figure 2) was custom-designed to hold the Narrative Clip in place at a specific angle. Participants are instructed to attach the Narrative Clip to the 3D printed wedge, which is attached to a band, and then wear it around their forehead. Participants are instructed to cover the camera lens or take off the Narrative Clip as needed (eg, when the participant is using the bathroom). Additional instructions are to: (1) not wear the Narrative Clip to school or while sleeping, (2) plug the Narrative Clip into the laptop at the end of each day using the USB cable provided (ie, automatically upload and charge the device) and (3) review and delete any photos that contain sensitive information.

We classify intensity of every cognitive activity recorded by the Narrative Clip into three ordinal categories (ie,
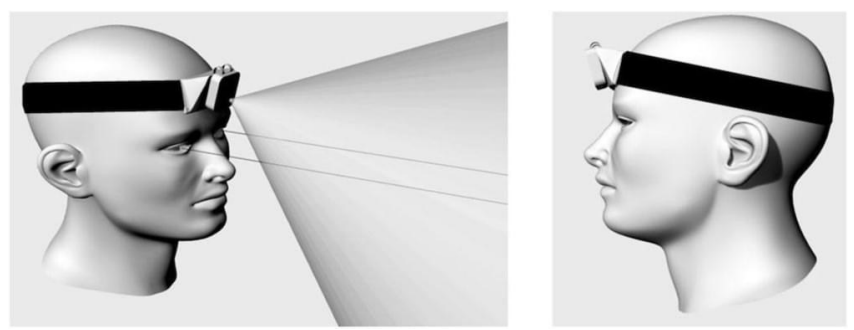

Figure 2 Narrative clip wedge. 
$1=$ low, $2=$ moderate and $3=$ high) according to published mental activity diary metrics. ${ }^{22}{ }^{29}$ Detailed descriptions of the procedure used to code intensity of cognitive activity is included in online supplementary appendix A. We also measure the duration of each of these activities in minutes based on the number of photos taken by Narrative Clip. The level of cognitive rest for each day is measured as the inverse of the summary score that sums the duration of various cognitive activities multiplied by the corresponding intensity of these cognitive activities (duration $\mathrm{x}$ intensity) across a whole day. Using established protocols, two trained coders will code the photo image data collected by the Narrative Clips for intensity and duration of each cognitive activity. Inter-rater reliability will be established before the coding. Any discrepancy in coding will be reviewed and discussed by the two coders until consensus is reached. Additionally, self-reported cognitive rest data are collected daily throughout study participation via the daily surveys and will be compared with the Narrative Clip data.

\section{Postconcussive symptoms}

Postconcussive symptoms are assessed daily by having youth complete the Post-Concussive Symptom Scale (PCSS) based on current symptoms. ${ }^{50}$ The PCSS consists of 22 symptoms ranging from 0 (no symptoms) to 6 (severe symptoms). The total PCSS score is the sum of the ratings (range $0-132$ ). The PCSS is the most commonly used concussion assessment tool among clinicians, with established reliability and normative data. ${ }^{50}$

\section{Pain}

Pain is self-reported daily by youth participants based on the severity of the pain they experienced on that given day using an 11-point numerical rating scale $(0=$ nopain to $10=$ worst pain possible).$^{51}$

\section{Variables and measures included in the three assessments \\ Functional outcomes}

Functional outcomes are evaluated during each postinjury assessment using the Functional Disability Inventory (FDI $)^{52}$ and Pediatric Quality of Life Inventory (PedsQL). ${ }^{53-55}$ Youth and his/her parent complete the FDI and PedsQL at all three time points to assess activity limitations attributable to the SRC, as well as the youth's overall quality of life. ${ }^{53-55}$ The FDI asks participants to rate perceived activity limitations due to physical health problems, while the PedsQL assesses broader aspects of quality of life, measured in terms of physical, emotional, social and school functioning. The PedsQL generates psychosocial and physical summary scores, as well as a total score. ${ }^{53-55}$ During the first assessment, retrospective ratings of preinjury functioning are obtained using the PedsQL.

\section{Potential confounders}

Premorbid child functioning

Premorbid child functioning is retrospectively rated by parents at the first assessment using the Behaviour
Assessment System for Youth, second edition. ${ }^{56}$ The Behavioural Symptoms Index provides a summary measure of premorbid behavioural adjustment, and the Adaptive Skills Composite score provides a summary measure of premorbid adaptive functioning.

\section{Concussion risk}

For the purposes of this study, we define a high-risk concussion as one surpassing an empirical threshold for acute symptoms on the PCSS. ${ }^{34} 3550$ Specifically, we will classify concussions with an acute total PCSS score of 13 or higher as high-risk. ${ }^{35}$

\section{Demographic information and postinjury events}

Demographic variables include age, sex, grade in school, sport, school, number of previous concussions, years of experience in the sport, prescribed rest, diet, device wear time and times from injury to return to school, start of the graded RTP protocol, completion of the RTP protocol and acute symptom resolution.

\section{Data management}

\section{Time-lapse photo-motion match data}

All ActiGraph and Narrative Clip data are automatically time and date stamped. After participants return study devices, data on postinjury physical and cognitive activity collected on the devices are transferred to a protected research server. Data on the protected server are only accessible to a designated subgroup of study staff, and are only accessible from systems connected internally to the organisation's network. We first use computer visualisation methods, including automatic extraction, analysis and image understanding, to process and analyse the metadata collected by the Narrative Clip (eg, total photographs taken, number of photographs deleted and time each photograph was taken). Custom developed software is then used to create time-coded, time-lapse photo-motion matched data based on the data collected from the Narrative Clip and ActiGraph, respectively (figure 3). Finally, we use algorithms to streamline analysis of the matched data (ie, Narrative Clip photograph and Actigraph motion) by identifying the matched data points with similar characteristics, which significantly reduces data processing and coding time. For example, 12 hours of images from the Narrative Clip (roughly 1440 photos) can be condensed to a $24 \mathrm{~s}$ long (30 frames per second, 1 minute time-lapse) video (figure 3). Using predefined criteria established by the research team (online supplementary appendix A), two trained research staff then review the photo-motion matched data to code and validate the intensity and duration of the cognitive activities captured by the Narrative Clip (described above).

\section{Missing data}

To handle missing data, we will first examine patterns of missing data and identify missing data mechanisms. We will then use approximate Bayesian Bootstrap or Markov Chain Monte Carlo methods in our main analyses if the assumption of missing completely at random or missing at random 


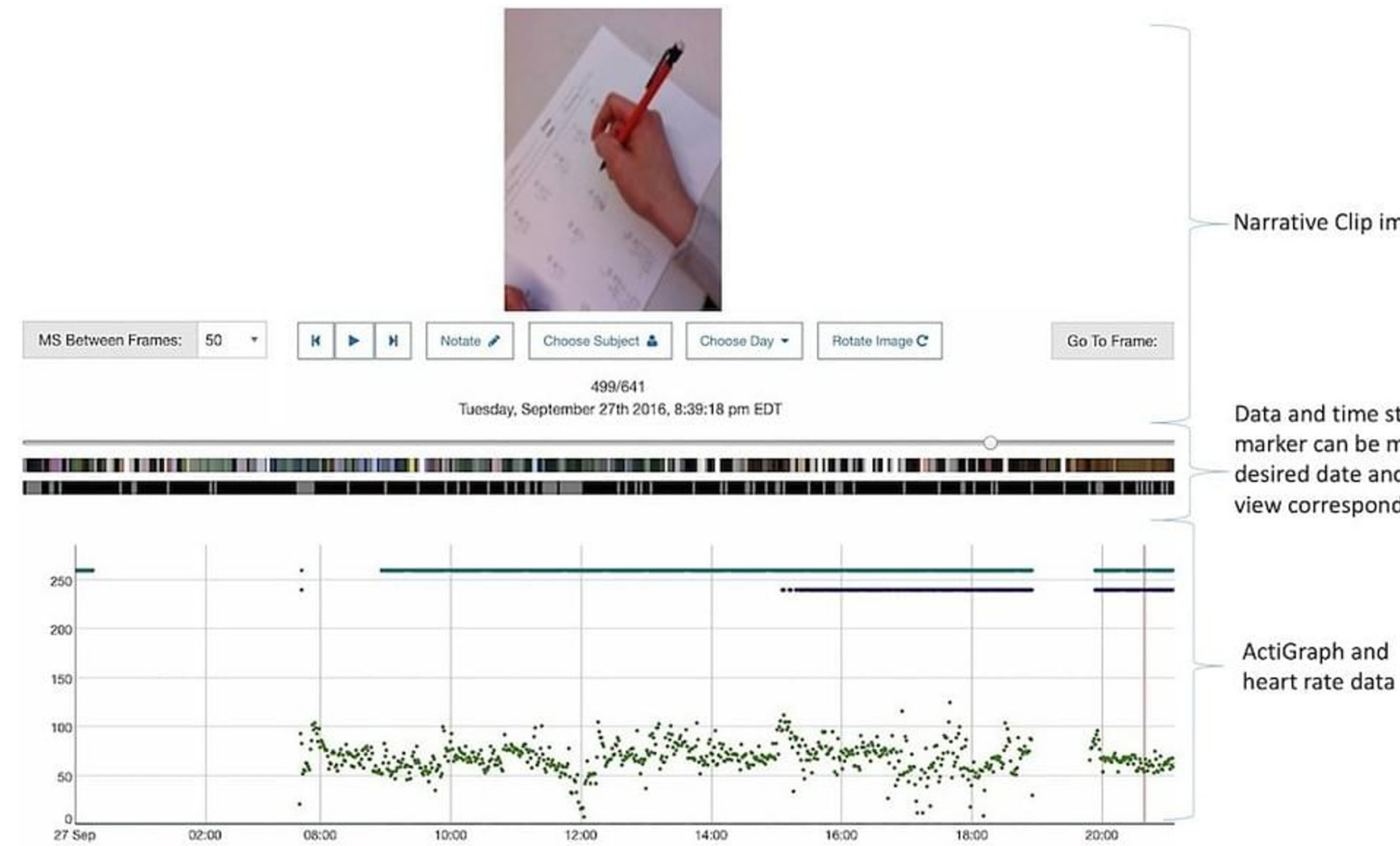

Figure 3 Time-lapse photo-motion matched data from the Narrative Clip and ActiGraph.

is met, respectively. We will use multiple imputation by fully conditional specification in our main analyses, followed by a sensitivity analysis if the assumption is violated. ${ }^{57}$

\section{Data analyses}

\section{Descriptive analyses}

We will describe the distributions of daily physical and cognitive rest by duration, intensity and levels (duration $\mathrm{x}$ intensity). We will report descriptive statistics and illustrate distributions of rest using Kernel density estimation. Comparisons will be made across subgroups, based on age, sex and concussion risk (high vs low) using analysis of variance or Kruskal-Wallis equality-of-populations rank test, as appropriate. We will compare the difference between rest type (physical vs cognitive) using paired t-test or Wilcoxon matched-pairs sign rank test. We will also examine the consistency between prescribed rest and actual rest, and objectively measured physical and cognitive rest and self-reported physical and cognitive rest, using Pearson correlation or Spearman rank correlation analysis.

\section{Primary analyses}

We will use Cox proportional hazards models to identify the duration, intensity and levels (duration $\mathrm{x}$ intensity) of physical and cognitive rest after SRC in relation to the number of days from injury to symptom resolution, censored or to the end of the study at day 45, whichever comes first. ${ }^{58}$ Propensity scores will be used to mimic the homogenous or balanced condition for high levels versus moderate levels, as well as low levels versus moderate levels, of physical and cognitive rest, respectively, accounting for all covariates, including demographics, sport and school characteristics, acute clinical variables, injury severity, premorbid conditions and pain. ${ }^{59}$ Using a novel approach ('restricted cubic splines' in the Cox models), ${ }^{60}$ we will first test whether the relationship between physical and cognitive rest and the outcome of interest are linear or non-linear. If a U-shaped or J-shaped (non-linear) dose-response relationship is found, we will then identify the optimal dosage (ie, duration, intensity or levels of physical and cognitive rest). We will assess the proportional hazards assumption with graphical methods, and by adding time-by-covariate interaction terms in the models. If the assumption of proportional hazards is violated, we will consider other alternatives, such as Poisson regression.

To identify the duration, intensity and postinjury levels of physical and cognitive rest in relation to continuous variables of functional outcomes, we will construct a 2-level linear mixed model with repeated outcome measures at the three assessments (level 1), nested in individuals (level 2) using a restricted maximum likelihood method. ${ }^{61}$ We will use the same restricted cubic splines approach and propensity score methods described above to evaluate the duration, intensity and levels of physical and cognitive rest.

\section{Secondary/exploratory analyses}

Similar analyses will be conducted to test whether the duration, intensity and levels (duration $\mathrm{x}$ intensity) of physical and cognitive rest vary between youth with 
high-risk and low-risk concussions. We will also test for interactions between concussion risk and levels of rest using a likelihood ratio test. If the interaction terms are statistically significant, we will report stratified results for youth with high-risk or low-risk concussions to illustrate differences in duration, intensity and postinjury levels of physical and cognitive rest. ${ }^{61}$

\section{Statistical power}

We calculated sample size and study power based on the log-rank test of two survival rates for simplicity. ${ }^{62}{ }^{63} \mathrm{We}$ define the date of symptom resolution as the event of interest, and divide the summary scores of physical and cognitive rest into three groups: (1) below, (2) within and (3) above the optimal levels of physical and cognitive rest. Using the second category as the reference group and considering the multiple comparisons across the three groups, we will apply a Bonferroni adjusted alpha level of $0.025[0.05 /(3-1)]$. Based on the results from our pilot study, we estimate that (1) $50 \%$ of participants are within the optimal level of rest and 95\% of these participants' symptoms will resolve within 28 days, and (2) $15 \%$ are above and $35 \%$ are below the optimal level of rest and $75 \%$ of these participants' symptoms will resolve within 28 days. With a total sample size of $n=110$ and one-sided significance test, we have $86.8 \%$ power to detect a $20 \%$ decrement in recovery rate comparing the below to within optimal level of rest group. If we have up to $10 \%$ loss to follow-up, we still have at least $83.2 \%$ power to detect a decrement of $20 \%$ in recovery rate.

\section{Patient and public involvement}

The study protocol and instruments used in this study have been successfully pilot tested with concussed children and their parents and demonstrated feasibility. Prior to obtaining consent/assent, we explain the objectives, expected benefits of the study and type of information collected to interested dyads. Informed written consent/ assent is then obtained from every participant. Data confidentiality is ensured and maintained throughout the study. The findings of this study (edited in lay language) will be disseminated to study participants through a newsletter. We will also disseminate our study findings at scientific conferences and by peer-reviewed publications.

\section{ETHICS}

No significant risks are associated with participation in the study, and participation does not affect the care provided to participants. Since participants are asked to wear a Narrative Clip that takes a picture every $30 \mathrm{~s}$, the research team has worked very closely with the Privacy and Security Advisory Committee at $\mathrm{NCH}$ to identify potential risks to privacy and confidentiality, and developed procedures and technical controls to mitigate the potential risks. These strategies include blocking all uploaded data from the Narrative Clip cloud service, disconnecting laptops from the internet, only storing images on the laptop, providing training to youth participants and their parent on proper device utilisation and photograph review, and establishing a 'chain of custody' for handling the laptop and devices. Further, youth are assured that only trained researchers will have access to the data.

\section{DISSEMINATION}

We will use common dissemination strategies to disseminate our study findings: (1) traditional academic outreach (eg, publications in peer-reviewed journals and presentations at professional conferences), (2) media outreach (eg, newspapers, radio, TV, social media) and the creation of related materials (eg, reports, special interest newsletters), (3) personal contacts (eg, professional networks), (4) key stakeholders and organisations (eg, youth sports leagues), and (5) creating actionable recommendations for concussion prevention programmes.

\section{DISCUSSION}

This prospective cohort study aims to objectively measure physical and cognitive rest following SRC and identify the level of rest that facilitates recovery and optimises postconcussion outcomes for youth with high-risk or low-risk SRCs. Engaging in high levels of activity too soon after concussion could exacerbate symptom duration and lead to greater neurocognitive and functional impairments. ${ }^{19-23}$ Conversely, a period of 'complete' rest, especially for long periods of time, could negatively affect youth with concussion because of muscular deconditioning and withdrawal from school and sport activities, and may also be impractical or unnecessary after some concussions. ${ }^{28-30}$ To the best of our knowledge, this study is the first of its kind to objectively measure and collect real-time data on the type, duration and intensity of physical and cognitive activities (and, conversely, rest) postconcussion. The study findings will therefore fill a critical knowledge gap, and will enable identification of the level of physical and cognitive rest needed following SRC to facilitate recovery based on concussion risk. ${ }^{31} 32$

The study has several limitations. First, quantifying cognitive rest is challenging. Although we document and track activities that require mental concentration using both objective and self-report data, we are not able to measure participants' actual mental engagement in each cognitive activity. Second, participants are instructed not to wear the Narrative Clip to school because of privacy concerns; thus, we are unable to document the cognitive activities that participants engage in during their time at school. Third, participants may opt out of wearing the devices, or their activities may not be recorded if they improperly wear the devices. Fourth, patient and injury characteristics may differ for youths recruited from local middle and high schools compared with those recruited from the ED.

Despite these limitations, the study will foster the development of better tools to objectively measure physical 
and cognitive rest postconcussion. The results of the study will help move us beyond the current clinical practice of 'one-size-fits-all' rest plans, ${ }^{34} 35$ and will provide evidencebased, individually tailored rest recommendations, which can be evaluated in a subsequent RCT. Finally, the results of the study will shape standards of care and inform treatment decisions about optimal levels of physical and cognitive rest for youth following SRC.

\section{Author affiliations}

${ }^{1}$ Center for Injury Research and Policy, The Research Institute at Nationwide Children's Hospital, Columbus, Ohio, USA

${ }^{2}$ Pediatrics, The Ohio State University, Columbus, Ohio, USA

${ }^{3}$ Psychology, University of Calgary, Calgary, Alberta, Canada

${ }^{4}$ Department of Epidemiology and Biostatistics, Indiana University, Bloomington, Indiana, USA

${ }^{5}$ Center for Biobehavioral Health, The Research Institute at Nationwide Children's Hospital, Columbus, Ohio, USA

${ }^{6}$ Sports Medicine, Nationwide Children's Hospital, Columbus, Ohio, USA

${ }^{7}$ Sport and Internal Medicine, The Ohio State University, Columbus, Ohio, USA

${ }^{8}$ Emergency Medicine, Nationwide Children's Hospital, Columbus, Ohio, USA

${ }^{9}$ Research Information Solution and Innovation, The Research Institute at

Nationwide Children's Hospital, Columbus, Ohio, USA

${ }^{10}$ Psychology, The Ohio State University, Columbus, Ohio, USA

Acknowledgements We would like to thank the following individuals for their assistance and contributions in the development of the custom developed software (Matthew Bailey) and the design of a 3D printed wedge (Robert Strouse). We acknowledge the invaluable contributions of the Nationwide Children's Hospital athletic trainers and ED research coordinators, as well as the student-athletes who were involved in this project.

Contributors JZY initiated and conceived the study, drafted the research protocol and critically revised the protocol. KOY and PCX participated in the study design and the protocol development in collaborations with JZY, and critically reviewed and revised the protocol. LS, BS and AN assisted in development of the recruitment procedures and critically revised the content of the written protocol. HGT and ZLL assisted in the selection of study measurement tools and critically revised the content of the written protocol. JM, TP, MT and DMC assisted in development of the data collection methods and critically reviewed and revised the written protocol. YGH and JP developed custom software and algorism to match the ActiGraph and Narrative Clip data and critically reviewed and revised the written protocol. All authors approved the final version of this protocol.

Funding This work was supported by Eunice Kennedy Shriver National Institute of Child Health and Human Development (NICHD) grant number [1R21HD086451-01A1].

Competing interests None declared.

Patient consent for publication Not required.

Ethics approval This study has received ethical approval from the Institutional Review Board (IRB) at the participating institution (IRB at NCH, IRB16-00613).

Provenance and peer review Not commissioned; externally peer reviewed.

Open access This is an open access article distributed in accordance with the Creative Commons Attribution Non Commercial (CC BY-NC 4.0) license, which permits others to distribute, remix, adapt, build upon this work non-commercially, and license their derivative works on different terms, provided the original work is properly cited, appropriate credit is given, any changes made indicated, and the use is non-commercial. See: http://creativecommons.org/licenses/by-nc/4.0/.

\section{REFERENCES}

1. Centers for Disease Control and Prevention. Nonfatal traumatic brain injuries related to sports and recreation activities among persons aged $\leq 19$ years--United States, 2001-2009. MMWR Morb Mortal Wkly Rep 2011;60:1337-42.

2. Bryan MA, Rowhani-Rahbar A, Comstock RD, et al. Sportsand recreation-related concussions in US Youth. Pediatrics 2016;138:e20154635.
3. Lincoln AE, Caswell SV, Almquist JL, et al. Trends in concussion incidence in high school sports: a prospective 11-year study. Am J Sports Med 2011;39:958-63.

4. Taylor HG, Dietrich A, Nuss K, et al. Post-concussive symptoms in children with mild traumatic brain injury. Neuropsychology 2010;24:148-59.

5. Yeates KO. Mild traumatic brain injury and postconcussive symptoms in children and adolescents. J Int Neuropsychol Soc 2010;16:953-60.

6. McCrory P, Meeuwisse WH, Aubry M, et al. Consensus statement on concussion in sport: the 4th International Conference on Concussion in Sport held in Zurich, November 2012. Br J Sports Med 2013;47:250-8.

7. Yeates KO, Kaizar E, Rusin J, et al. Reliable change in postconcussive symptoms and its functional consequences among children with mild traumatic brain injury. Arch Pediatr Adolesc Med 2012;166:615-22.

8. Halstead ME, Walter KD. Council on Sports Medicine and Fitness. American Academy of Pediatrics. Clinical report--sportrelated concussion in children and adolescents. Pediatrics 2010;126:597-615

9. McCrea M, Guskiewicz KM, Marshall SW, et al. Acute effects and recovery time following concussion in collegiate football players: the NCAA Concussion Study. JAMA 2003;290:2556-63.

10. Meehan WP. 3rd, d'Hemecourt P, Comstock RD. High school concussions in the 2008-2009 academic year: mechanism, symptoms, and management. Am J Sports Med 2010;38:2405-9.

11. McCrory P, Meeuwisse W, Aubry M, et al. Consensus statement on Concussion in Sport - The 4th International Conference on Concussion in Sport held in Zurich, November 2012. Phys Ther Sport 2013;14:e1-13.

12. Lumba-Brown A, Yeates KO, Sarmiento K, et al. Centers for Disease Control and Prevention Guideline on the Diagnosis and Management of Mild Traumatic Brain Injury Among Children. JAMA Pediatr $2018 ; 172$.

13. Lumba-Brown A, Yeates KO, Sarmiento $K$, et al. Diagnosis and Management of Mild Traumatic Brain Injury in Children: A Systematic Review. JAMA Pediatr 2018;172.

14. Grady MF, Master CL, Gioia GA. Concussion pathophysiology: rationale for physical and cognitive rest. Pediatr Ann 2012;41:377-82

15. Giza CC, Hovda DA. The Neurometabolic Cascade of Concussion. J Athl Train 2001;36:228-35.

16. Griesbach GS, Gómez-Pinilla F, Hovda DA. Time window for voluntary exercise-induced increases in hippocampal neuroplasticity molecules after traumatic brain injury is severity dependent. $J$ Neurotrauma 2007;24:1161-71.

17. Maugans TA, Farley $\mathrm{C}$, Altaye $\mathrm{M}$, et al. Pediatric sports-related concussion produces cerebral blood flow alterations. Pediatrics 2012;129:28-37.

18. Udomphorn Y, Armstead WM, Vavilala MS. Cerebral blood flow and autoregulation after pediatric traumatic brain injury. Pediatr Neurol 2008;38:225-34.

19. Majerske CW, Mihalik JP, Ren D, et al. Concussion in sports: postconcussive activity levels, symptoms, and neurocognitive performance. J Athl Train 2008;43:265-74.

20. Moser RS, Glatts C, Schatz P. Efficacy of immediate and delayed cognitive and physical rest for treatment of sports-related concussion. J Pediatr 2012;161:922-6.

21. Gibson S, Nigrovic LE, O'Brien M, et al. The effect of recommending cognitive rest on recovery from sport-related concussion. Brain Inj 2013;27(7-8):839-42.

22. Brown NJ, Mannix RC, O'Brien MJ, et al. Effect of cognitive activity level on duration of post-concussion symptoms. Pediatrics 2014;133:e299-304.

23. Moser RS, Schatz P. A Case for Mental and Physical Rest in Youth Sports Concussion: It's Never too Late. Front Neurol 2012;3:171.

24. Howell DR, Mannix RC, Quinn B, et al. Physical activity level and symptom duration are not associated after concussion. Am J Sports Med 2016;44:1040-6.

25. Leddy JJ, Baker JG, Willer B. Active rehabilitation of concussion and post-concussion syndrome. Phys Med Rehabil Clin N Am 2016;27:437-54.

26. Lal A, Kolakowsky SA, et al. The effect of physical exercise after a concussion. AJSM 2018;46:743-52.

27. Grool AM, Aglipay M, Momoli F, et al. Association between early participation in physical activity following acute concussion and persistent postconcussive symptoms in children and adolescents. JAMA 2016;316:2504-14.

28. Thomas DG, Apps JN, Hoffmann RG, et al. Benefits of strict rest after acute concussion: a randomized controlled trial. Pediatrics $2015 ; 135$. 
29. Kirkwood MW, Randolph C, Yeates KO. Sport-related concussion: a call for evidence and perspective amidst the alarms. Clin J Sport Med 2012;22:383-4

30. Meehan WP, Bachur RG. The recommendation for rest following acute concussion. Pediatrics 2015;135:362-3

31. Graham R, Rivara FP, Ford MA, et al. Sport related concussion in youth: improving the science, changing the culture. Washington, DC 2014.

32. McCrea M, Broshek DK, Barth JT. Sports concussion assessment and management: future research directions. Brain Inj 2015;29:276-82.

33. Arbogast KB, McGinley $A D$, Master $C L$, et al. Cognitive rest and school-based recommendations following pediatric concussion: the need for primary care support tools. Clin Pediatr 2013;52:397-402.

34. McCrea M, Guskiewicz K, Randolph C, et al. Incidence, clinica course, and predictors of prolonged recovery time following sport-related concussion in high school and college athletes. J Int Neuropsychol Soc 2013;19:22-33.

35. Meehan WP, Mannix R, Monuteaux MC, et al. Early symptom burden predicts recovery after sport-related concussion. Neurology 2014;83:2204-10.

36. Adelson PD, Pineda J, Bell MJ, et al. Common data elements for pediatric traumatic brain injury: recommendations from the working group on demographics and clinical assessment. J Neurotrauma 2012;29:639-53.

37. McCrory P, Feddermann-Demont N, Dvořák J, et al. What is the definition of sports-related concussion: a systematic review. $\mathrm{Br} J$ Sports Med 2017;51:877-87.

38. McCrea MKJ, Randolph C. Standardized Assessment of Concussion (SAC): manual for administration, scoring, and interpretation. 3rd edn. Waukesha, Wl: Comprehensive Neuropsychological Services, 2000.

39. McCrea M. Standardized Mental Status Testing on the Sideline After Sport-Related Concussion. J Athl Train 2001;36:274-9.

40. Giza CC, Kutcher JS, Ashwal S, et al. Summary of evidence-based guideline update: evaluation and management of concussion in sports: report of the Guideline Development Subcommittee of the American Academy of Neurology. Neurology 2013;80:2250-7.

41. Collie A, Maruff P, Makdissi M, et al. CogSport: reliability and correlation with conventional cognitive tests used in postconcussion medical evaluations. Clin J Sport Med 2003;13:28-32.

42. CogState L. CogSport [Computer software]. Parkville, Victoria, Australia, 1999.

43. Collie A, Darby D, Maruff P. Computerised cognitive assessment of athletes with sports related head injury. $\mathrm{Br} J$ Sports Med 2001;35:297-302

44. Guskiewicz KM. Postural stability assessment following concussion: one piece of the puzzle. Clin J Sport Med 2001;11:182-9.

45. Martin JL, Hakim AD. Wrist actigraphy. Chest 2011;139:1514-27.

46. Bouchard C, Tremblay A, Leblanc C, et al. A method to assess energy expenditure in children and adults. Am J Clin Nutr 1983;37:461-7.
47. Wiebe DJ, Nance ML, Houseknecht E, et al. Ecologic momentary assessment to accomplish real-time capture of symptom progression and the physical and cognitive activities of patients daily following concussion. JAMA Pediatr 2016;170:1108-10.

48. Berger I, Obeid J, Timmons BW, et al. Exploring accelerometer versus self-report sleep assessment in youth with concussion. Glob Pediatr Health 2017:4:1-9.

49. Narrative Clip-A new kind of photographic memory. 2014 http:// getnarrative.com/.

50. Joyce AS, Labella CR, Carl RL, et al. The Postconcussion Symptom Scale: utility of a three-factor structure. Med Sci Sports Exerc 2015;47:1119-23.

51. von Baeyer CL, Spagrud LJ, McCormick JC, et al. Three new datasets supporting use of the Numerical Rating Scale (NRS-11) for children's self-reports of pain intensity. Pain 2009;143:223-7.

52. Claar RL, Walker LS. Functional assessment of pediatric pain patients: psychometric properties of the functional disability inventory. Pain 2006;121(1-2):77-84.

53. Varni JW, Limbers CA. The pediatric quality of life inventory: measuring pediatric health-related quality of life from the perspective of children and their parents. Pediatr Clin North Am 2009;56:843-63.

54. Varni JW, Burwinkle TM, Seid M, et al. The PedsQL 4.0 as a pediatric population health measure: feasibility, reliability, and validity. Ambul Pediatr 2003;3:329-41.

55. McCarthy ML, MacKenzie EJ, Durbin DR, et al. The pediatric quality of life inventory: an evaluation of its reliability and validity for children with traumatic brain injury. Arch Phys Med Rehabil 2005;86:1901-9.

56. Yeates KO, Taylor HG, Rusin J, et al. Premorbid child and family functioning as predictors of post-concussive symptoms in children with mild traumatic brain injuries. Int J Dev Neurosci 2012;30:231-7.

57. Little RJA, Rubin DB. Statistical analysis of missing data. New York: Wiley, 2002.

58. Andersen PK, Klein JP, Knudsen KM, et al. Estimation of variance in Cox's regression model with shared gamma frailties. Biometrics 1997:53:1475-84.

59. Angrist JD, Kuersteiner GM. Semiparametric causality tests using the policy propensity score. Cambridge, MA: National Bureau of Economic Research, 2004.

60. Desquilbet L, Mariotti F. Dose-response analyses using restricted cubic spline functions in public health research. Stat Med 2010;29:1037-57.

61. Proust-Lima C, Dartigues JF, Jacqmin-Gadda $H$. Misuse of the linear mixed model when evaluating risk factors of cognitive decline. Am J Epidemiol 2011;174:1077-88.

62. Freedman LS. Tables of the number of patients required in clinical trials using the logrank test. Stat Med 1982;1:121-9.

63. Maas CJM, Hox JJ. Sufficient sample sizes for multilevel modeling Methodology 2005;1:86-92. 